\title{
Energetic Geodesic Acoustic Modes Associated with Two-Stream-like Instabilities in Tokamak Plasmas
}

\author{
Z. S. Qu* and M. J. Hole \\ Research School of Physics and Engineering, The Australian National University, \\ Canberra, Australian Capital Territory 2601, Australia \\ M. Fitzgerald \\ CCFE Fusion Association, Culham Science Centre, Abingdon, Oxon OX14 3DB, United Kingdom
}

(Received 26 November 2015; published 4 March 2016)

\begin{abstract}
An unstable branch of the energetic geodesic acoustic mode (EGAM) is found using fluid theory with fast ions characterized by their narrow width in energy distribution and collective transit along field lines. This mode, with a frequency much lower than the thermal GAM frequency $\omega_{\mathrm{GAM}}$, is now confirmed as a new type of unstable EGAM: a reactive instability similar to the two-stream instability. The mode can have a very small fast ion density threshold when the fast ion transit frequency is smaller than $\omega_{\mathrm{GAM}}$, consistent with the onset of the mode right after the turn-on of the beam in DIII-D experiments. The transition of this reactive EGAM to the velocity gradient driven EGAM is also discussed.
\end{abstract}

DOI: 10.1103/PhysRevLett.116.095004

Recent experiments [1-3] with neutral beam injection show bursting $n=0$ axisymmetric modes at half of the thermal geodesic acoustic mode (GAM) [4,5] frequency, which are identified as the energetic-particle-induced GAMs (EGAMs). The presence of EGAMs is found responsible for fast ion losses [6] and may enhance turbulence transport, leading to the destruction of internal transport barriers [7] and the degradation of fusion confinement. Many efforts have been made to model [8-12] and simulate [13-16] EGAMs both linearly and nonlinearly using a kinetic or hybrid-kinetic theory. One of the major outcomes is the discovery of multiple branches of GAMs in the presence of fast particles. The lower frequency branch is excited by the inverse Landau damping provided by the fast ions. In tokamaks, most of these works assume a fast ion distribution with a large width in energy (e.g., the slowingdown distribution function). However, the magnetic spectrogram in DIII-D experiments [1] showed a turn-on of the mode $1 \mathrm{~ms}$ right after the beam switched on, much faster than the beam slowing-down time (approximately tens of milliseconds), indicating that the beam ions are not slowed down when the mode first appears. Also, due to the limited width in energy distribution, the beam may not provide sufficient inverse Landau damping to enable the growth of the mode. One possible explanation is proposed by Berk and Zhou [9], in which the early EGAMs are negative energy modes, the presence of which will reduce the total energy of the system. They are driven unstable by fast ion Landau damping.

Because of its simplicity and intuitive nature, the fluid theory, if its regime of validity is properly considered, may shed light on the underlying physics which may otherwise be confused with wave-particle interaction physics. By using a fluid description of the fast ions, we have found a new class of unstable EGAMs associated with beam ions. These EGAMs are similar to the two-stream instabilities rather than driven by the inverse Landau damping. They have a high growth rate ( $\sim 30 \%$ mode frequency), which increases steeply as fast ion density increases, consistent with the early turn-on of the mode.

We consider a tokamak plasma with large aspect ratio, circular cross section, and low $\beta$. The flux surfaces are concentric and labeled by radial coordinate $r$, while $\theta$ and $\varphi$ give the poloidal and toroidal angle, respectively. In this work, we adopt a local treatment, making $\rho_{s}<q \rho_{s} \ll$ $L_{\mathrm{EGAM}}$, where $L_{\mathrm{EGAM}}$ is the width of the mode, $\rho_{s}$ the Larmor radius, and $q \rho_{s}$ gives approximately the drift orbit width. The change of equilibrium quantities in the radial direction is ignored. We assume that the plasma consists of thermal and fast ions, all with mass $m_{i}$ and unity charge $e$, as well as electrons with negligible inertia and negative charge $-e$. Thermal ions have density $n_{i}$ and temperature $T_{i}$, while for fast ions, the density $n_{f}$, the parallel pressure $p_{\| f}$, and the perpendicular pressure $p_{\perp f}$ are obtained by integrals of the fast ion guiding center distribution. The thermal ions are static with $V_{i}=0$. The fast ions have an average transit speed $V_{f}$ along the field lines.

The dynamics of the system is determined by the linearized momentum equation of each species " $s$," given by

$$
\begin{aligned}
& m_{s} n_{s}\left(\frac{\partial \tilde{\boldsymbol{V}}_{s}}{\partial t}+\frac{\tilde{n}_{s}}{n_{s}} \boldsymbol{V}_{s} \cdot \nabla \boldsymbol{V}_{s}+\boldsymbol{V}_{s} \cdot \nabla \tilde{\boldsymbol{V}}_{s}+\tilde{\boldsymbol{V}}_{s} \cdot \nabla \boldsymbol{V}_{s}\right) \\
& =n_{s} q_{s}\left(-\nabla \tilde{\Phi}+\tilde{\boldsymbol{V}}_{s} \times \boldsymbol{B}\right)-\nabla \cdot \tilde{\overline{\mathrm{P}}}
\end{aligned}
$$


in which $q_{s}$ is the charge, $\tilde{\Phi}$ the perturbed electrostatic potential, $\tilde{\overline{\mathrm{P}}}=\tilde{p_{\perp}} \bar{I}+\left(\tilde{p_{\|}}-\tilde{p_{\perp}}\right) \boldsymbol{b} \boldsymbol{b}$ the perturbed pressure tensor, with $\boldsymbol{b}=\boldsymbol{B} / \boldsymbol{B}$, and $\boldsymbol{B}$ the magnetic field. The subscript $s$ labels electrons $(e)$, thermal ions $(i)$, or fast ions $(f)$, and the circumflex labels the perturbed quantities. The perturbed velocity consists of the perpendicular and parallel components, written as

$$
\tilde{\boldsymbol{V}}_{s}=\tilde{\boldsymbol{V}}_{E}+\left[\tilde{V}_{s+}(r) e^{i \theta}+\tilde{V}_{s-}(r) e^{-i \theta}\right] \boldsymbol{b},
$$

where $\tilde{\boldsymbol{V}}_{E}$ is the $\boldsymbol{E} \times \boldsymbol{B}$ drift velocity. Considering the small orbit width assumption, we only retain the $m=0$ component of $\tilde{\Phi}$ for the $\boldsymbol{E} \times \boldsymbol{B}$ drift and $m= \pm 1$ components of parallel velocity that are lowest order in $q \rho_{s}$, while the magnetic gradient or curvature drifts are higher order terms and therefore ignored. Now $\tilde{\boldsymbol{V}}_{E}$ is in the direction of $\boldsymbol{\pi}=\boldsymbol{e}_{r} \times \boldsymbol{b}$. Similarly, the perturbed density and pressure are decomposed into $m= \pm 1$ harmonics; for instance, $\tilde{n}_{i}=\tilde{n}_{i+1} e^{i \theta}+\tilde{n}_{i-1} e^{-i \theta}$.

The ion response to $\tilde{\boldsymbol{V}}_{E}$ is described by the ChewGoldberger-Low (CGL) law [17], assuming that the ion perpendicular and parallel pressure are doing work independently. An agreement is reached between the CGL law and the gyrokinetic theory on the thermal GAM frequency $[18,19]$. It has also been shown that when the mode frequency is much higher than the thermal frequency of the bulk ions (in conventional GAM, $q \gg 1$ ), the CGL law can give a good description of the plasma response [20,21]. This CGL law is given by

$$
\begin{gathered}
\frac{d p_{\|_{s}}}{d t}=-p_{\|_{s}} \nabla \cdot \tilde{\boldsymbol{V}}_{s}-2 p_{\|_{s}} \boldsymbol{b} \cdot\left(\boldsymbol{b} \cdot \tilde{\boldsymbol{V}}_{s}\right), \\
\frac{d p_{\perp s}}{d t}=-2 p_{\perp s} \nabla \cdot \tilde{\boldsymbol{V}}_{s}+p_{\perp s} \boldsymbol{b} \cdot\left(\boldsymbol{b} \cdot \tilde{\boldsymbol{V}}_{s}\right) .
\end{gathered}
$$

The response of the electrons is assumed to be isothermal, which means

$$
\tilde{p}_{e}=\tilde{n}_{e} T_{e}=\left(\tilde{n}_{i}+\tilde{n}_{f}\right) T_{e},
$$

from the quasineutrality condition, while $\tilde{n}_{i}$ and $\tilde{n}_{f}$ are obtained from the ion continuity equation given by

$$
\frac{\partial \tilde{n}_{s}}{\partial t}=-\nabla \cdot\left(n_{s} \tilde{\boldsymbol{V}}_{s}+\tilde{n}_{s} \boldsymbol{V}_{s}\right) .
$$

We can simplify Eqs. (3), (4), and (6) using the identity

$$
\nabla \cdot \boldsymbol{\pi} \approx-\boldsymbol{\pi} \cdot \nabla \ln B \approx(\boldsymbol{b} \cdot \nabla \boldsymbol{\pi}) \cdot \boldsymbol{b}=-\kappa_{g} \approx-\sin \theta / R,
$$

which are all considered as geodesic curvature, giving the relationship between the perturbed pressure and perturbed velocity. We substitute these relationships into Eq. (1) to eliminate the perturbed pressures.
Adding up Eq. (1) for electrons, thermal and fast ions, ignoring electron inertia, imposing the quasineutrality condition $\nabla \cdot \tilde{\boldsymbol{J}}=0(\tilde{\boldsymbol{J}}$ is the perturbed current), and taking a flux surface average, we obtain the perpendicular momentum equation. The parallel momentum equations for thermal and fast ions are obtained from the parallel component of Eq. (1), with the potential terms canceled using the same equation for electrons. These equations close the system and define the dispersion relationship $D(\omega)=0$, where $\omega$ is the mode complex angular frequency $[\gamma=\operatorname{Im}(\omega)$ gives the growth rate]. In the $q \gg 1$ limit, where the coupling to the thermal ion sound wave is ignored, $D(\omega)$ is given by

$$
D(\omega)=1-(1-\alpha) \frac{\omega_{\mathrm{GAM}}^{2}}{\omega^{2}}-\alpha G(\omega),
$$

where $\omega_{\mathrm{GAM}}^{2}=\left(2 T_{i} / m_{i} R^{2}\right)\left[(7 / 4)+\left(T_{e} / T_{i}\right)+O\left(1 / q^{2}\right)\right]$ is the square of the thermal GAM frequency and $\alpha \equiv$ $\left\langle n_{f}\right\rangle / n_{\text {total }}$ is the fast population fraction. The exact form of $G(\omega)$ depends on the fast ion distribution function, but since the fluid theory has ignored Landau damping effects, $G(\omega)$ is real if $\operatorname{Im}(\omega)=0$.

We first consider a bump-on-tail distribution function given by

$$
F\left(v_{\|}, v_{\perp}\right)=n_{f} A \exp \left[-\frac{m_{i}\left(v_{\|}-V_{f}\right)^{2}+m_{i} v_{\perp}^{2}}{2 T_{f}}\right],
$$

where $A$ is a normalization factor and $v_{\|}$and $v_{\perp}$ are parallel and perpendicular velocity, respectively. For small $\alpha$ and negligible energy width $T_{f}, G(\omega)$ is given by

$$
G(\omega) \approx \frac{\frac{3}{2} \omega_{b}^{2} q^{2}}{\omega^{2}-\omega_{b}^{2}}+\frac{\omega_{b}^{4} q^{2}}{\left(\omega^{2}-\omega_{b}^{2}\right)^{2}},
$$

where $\omega_{b} \equiv V_{f} / q R$ is the average fast ion transit frequency. The dispersion relationship now becomes a cubic equation of $\omega^{2}$ with three solutions. Properties of the solutions are determined by the relationship between $\omega_{\mathrm{GAM}}$ and $\omega_{b}$, as well as $q$ and the fast ion population.

In Fig. 1, we plot the solutions to $\omega$ with parameters $q=4$ and $\omega_{b}=0.58 \omega_{\mathrm{GAM}}$, retaining finite energy width $T_{f}=0.25 T_{i}$, for fast particle concentration from $1 \%$ to 40\%. Similar to Fu [8], multiple branches of GAMs are present. The frequency of the upper (frequency) EGAM, as seen in Fig. 1(a), increases with increasing fast particle population. This branch reduces to the thermal GAM when $n_{f}=0$. We compare our fluid result to the numerical solution of the kinetic dispersion relationship [16,22], showing very good agreement. In this case, the Landau damping from the thermal particles is negligibly small. Also, two complex conjugated branches are present in Figs. 1(b) and 1(d) at lower frequency, both having decreasing frequency with a higher fast ion fraction. We 

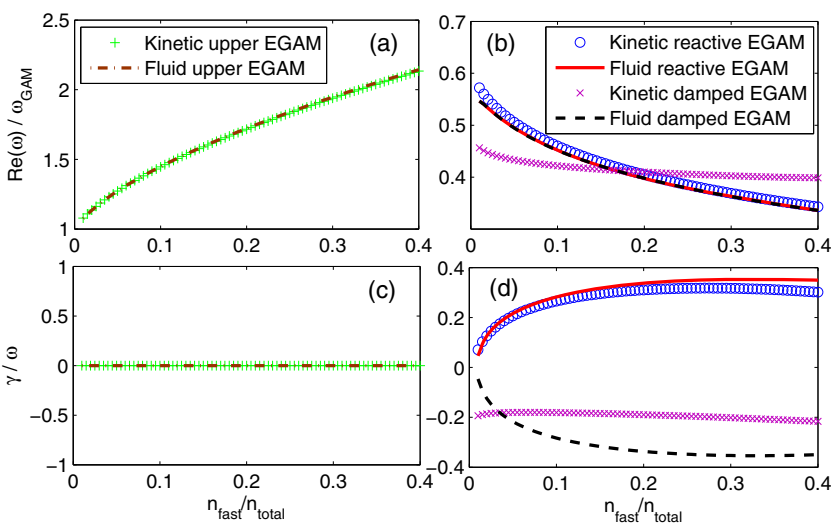

FIG. 1. Real frequency (a),(b) and growth rate (c),(d) versus fast ion density for multifluid model with comparison to kinetic theory, for $q=4$ and $\omega_{b}=0.58 \omega_{\mathrm{GAM}}$. Lines (symbols) represent fluid (kinetic) results.

note that the existence of these branches is due to the Doppler shift of the wave in the static frame of the fast ions, since this is the only effect of the fast ion when $T_{f}$ is small. One of these modes is found unstable in kinetic theory, and was previously attributed to the inverse Landau damping. However, the same growth rate is also obtained using the fluid model. Given that no Landau damping is present in the fluid theory, this instability cannot come from the waveparticle interaction, but must be a reactive instability. We name this unstable branch the reactive EGAM from its nature of instability. By solving the dispersion relationship Eqs. (8) and (10), we obtain the growth rate for $\alpha \ll 1$, given by

$$
\gamma \approx \frac{1}{2} q \omega_{b}^{2}\left(\omega_{\mathrm{GAM}}^{2}-\omega_{b}^{2}\right)^{-1 / 2} \sqrt{\alpha}
$$

with no fast ion density threshold. Finally, we find that the fluid theory is valid for the upper EGAM and the reactive EGAM, which are on the real axis or the upper plane, while the other damped EGAM is located on the lower plane and is strongly modified by Landau damping, leading to the deviation of its fluid solution from the kinetic theory.

For the regime $\omega_{b}>\omega_{\mathrm{GAM}}$, the upper EGAM will start at $\omega_{b}$ instead of the thermal GAM frequency, as shown in Fig. 2(a) with parameters $T_{f}=T_{i}, q=2$, and $\omega_{b}=1.76 \omega_{\mathrm{GAM}}$. The kinetic theory gives a finite Landau damping rate, while in the fluid theory this mode is predicted to be stable. One of the lower modes starts at $\omega_{\mathrm{GAM}}$, when $n_{f}=0$. Unlike Fig. 1, the lower modes have an instability threshold of $\alpha>0.05$. In Figs. 2(b) and 2(d), the unstable reactive EGAM and a damped EGAM occur between $0.05<\alpha<0.25$ when the two modes have the same real frequency. This real frequency bifurcates at $\alpha=0.25$, with the modes becoming stable at the same time. A parameter scan shows that the two bifurcation points move closer to each other when $\omega_{b}$ increases. For

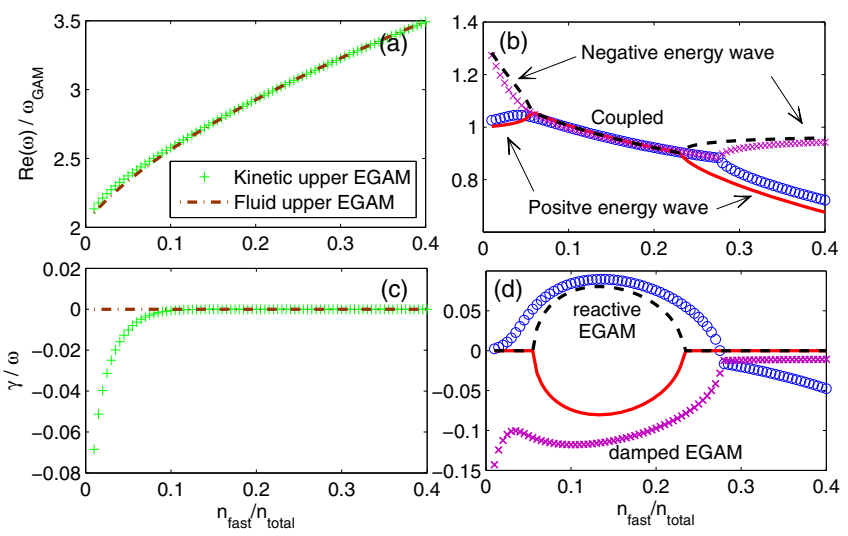

FIG. 2. Real frequency (a),(b) and growth rate (c),(d) versus fast ion density for multifluid model with comparison to kinetic theory, for $q=2$ and $\omega_{b}=1.76 \omega_{\mathrm{GAM}}$. Lines (symbols) represent fluid (kinetic) results.

$\omega_{b}>2 \omega_{\mathrm{GAM}}$, the two bifurcation points merge and no unstable mode is present for $0<\alpha<0.4$. Again, Fig. 2 shows a good match to the kinetic theory.

The origin of the instability can be studied by calculating the wave energy of the two lower frequency modes. In Fig. 2(b), when $\alpha>0.25$, the lower frequency mode is a positive energy wave $[d D(\omega) / d \omega>0]$, while the other is a negative energy wave $[d D(\omega) / d \omega<0]$. The strong coupling of these two modes is achieved when they possess the same real frequency $(0.05<\alpha<0.25)$, where the reactive EGAM occurs. The energy can transfer from the negative energy wave to the positive energy wave, enabling the growth of both modes meanwhile conserving the total energy [23]. Therefore, this GAM instability shares great similarities to the two-stream instabilities in a beam plasma system [24,25], which can also be captured by a fluid model.

We now study the relationship between the reactive EGAMs and the inverse Landau damping driven EGAMs (dissipative EGAMs). The unstable EGAM frequency and growth rate versus the fast particle energy width $T_{f}$ is plotted in Fig. 3 for the bump-on-tail distribution. The parameters are identical to Fig. 1. Figure 3 shows that the

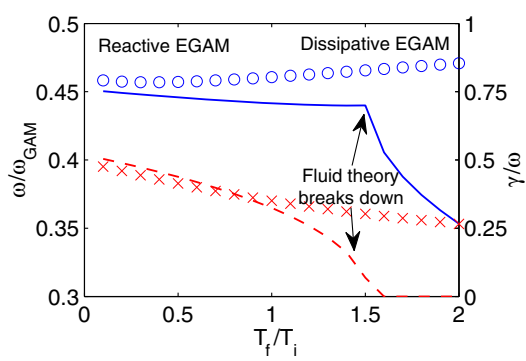

FIG. 3. Real frequency in fluid (blue solid line) and kinetic (blue circle) theory, and growth rate in fluid (red broken line) and kinetic (red cross) theory versus fast particle energy width $T_{f}$, for $n_{f} / n_{\text {total }}=0.1, q=4$, and $\omega_{b}=0.58 \omega_{\mathrm{GAM}}$. 
unstable EGAMs are reactive for $T_{f} / T_{i}<1$, where the fluid theory is valid, and dissipative for large $T_{f}$, where the kinetic effects are dominant and the fluid treatment breaks down. A smooth transition is found in between these two regimes by solving the kinetic dispersion relationship $[16,22]$. The conditions required for finding a reactive EGAM are given by

$$
\left|\omega-\omega_{b}\right|>\frac{1}{q R}\left(\frac{2 T_{f}}{m_{i}}\right)^{1 / 2} .
$$

One can use Eq. (11) and $\left|\omega-\omega_{b}\right| \approx \gamma$ for an estimation if $\omega_{\mathrm{GAM}}>\omega_{b}$. Further study shows that the smooth transition behavior is distribution shape independent.

Again, we have found great similarity between the EGAMs and the two-stream instabilities. It is well known that in a beam plasma system, for a single energy beam, the purely reactive two-stream instability occurs. On the other hand, if the bump slope is gentle, i.e., the beam is warm, the dissipative bump-on-tail instability occurs. With a finite but small beam energy width, the kinetic effects influence the reactive instability by breaking the complex conjugacy of the two fluid modes [26]. A change in the topology of the dispersion relationship is found as the beam thermal spread further increases, after which the dissipative drive becomes dominant [27]. These statements are also applicable to EGAMs.

Finally, we apply our results to the early turn-on of EGAMs in DIII-D by considering a single energy single pitch beam distribution function, before the slowing-down or pitch angle scattering can happen. This distribution function is given by

$$
F(E, \Lambda)=\frac{m_{i}^{2}\left|v_{\|}\right| n_{f}}{2 \pi E} \delta\left(E-E_{0}\right) \delta\left(\Lambda-\Lambda_{0}\right),
$$

where $E$ is the fast ion energy, $\Lambda$ the pitch angle, and $\delta(x)$ the Dirac delta function. We note that, in reality, the ionized beam will have a finite pitch angle spread and a first orbit loss for counterinjection. Additional studies show that as long as the width of the pitch angle distribution $\Delta \Lambda \ll \Lambda$ the pitch angle itself, our fluid theory will remain valid. The inclusion of $\Delta \Lambda$ will not change the result significantly as compared to the one assuming a single pitch angle. The form of $G(\omega)$ is identical to Eq. (10) except the numerators now become a function of both $E_{0}$ and $\Lambda_{0}$. For the DIII-D beam in Nazikian et al. [1], we have $E_{0}=75 \mathrm{keV}$ and $\Lambda_{0}=0.5$. We also have $\omega_{b}=0.88 \omega_{\mathrm{GAM}}$ obtained from $T_{e}=1.2 T_{i} \approx 1.2 \mathrm{keV}$ and $q=4$ at the radial localized flux surface $s=0.4$. Similarly, we plot the real frequency and growth rate of the reactive EGAM as a function of $\alpha$ in Fig. 4 (the other two branches are damped and not discussed here). The frequency of the reactive EGAM stays reasonably close to the observed frequency $(28 \mathrm{kHz})$ for $\alpha>3 \%$. Also, no density threshold is present in the fluid theory, although in reality the background damping

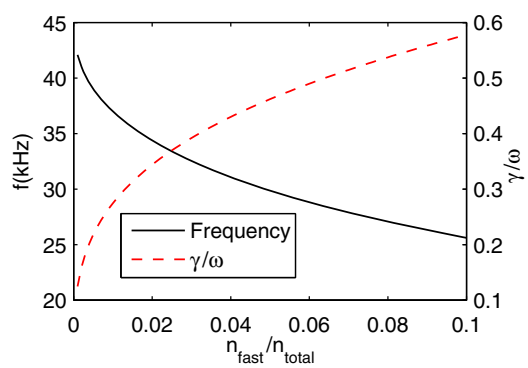

FIG. 4. Real frequency and growth rate of the reactive EGAM using DIII-D parameters on flux surface $s=0.4$.

(such as collisional damping) may create a finite threshold. But since the growth rate is large and is a steep function of the fast ion density when the density is low $(\sim \sqrt{\alpha})$, this background damping can be overcome quickly as fast ion density increases, consistent with the early turn-on of the mode. Furthermore, the smooth transition between reactive and dissipative EGAMs indicates the natural conversion from the early turn-on reactive EGAMs to the dissipative EGAMs, when the fast ions are slowed down in background plasma. Slowing-down of the fast ions due to the nonlinear phase of the reactive EGAMs is also possible and requires further investigation.

In summary, we have found a new unstable branch of EGAMs in the presence of beam ions with a small width in energy distribution, known as a reactive instability similar to the two-stream instability. This mode can have a much lower frequency than the thermal GAMs and $\gamma / \omega$ up to $30 \%$ with no turn-on threshold when background damping is not considered. Our work shows that EGAM solutions are not inherent to kinetic approaches and one should not overlook the reactive contribution to the instabilities. A smooth transition from reactive EGAMs to kinetic instabilities happens after the beam ions are slowed down, similar to the transition between the two-stream and bumpon-tail instabilities. We have also demonstrated the consistency of reactive EGAMs with the early turn-on of EGAMs in DIII-D experiments, a scenario that cannot be explained by the previous theories of inverse Landau damping driven EGAMs. In addition, this work gives a good example of how the fluid theory can aid the understanding of fast particle physics. Further discussion about the radial mode structure will be presented in future publications.

The authors would like to thank Dr. A. Biancalani, Dr. G. Y. Fu, Professor B. N. Breizman, and Dr. B. Layden for fruitful discussions, and Dr. M. A. Van Zeeland and Dr. R. Nazikian for DIII-D data.This work is funded by China Scholarship Council, and Australian ARC Projects No. DP1093797 and No. FT0991899. The authors would like to thank AINSE Ltd for providing financial assistance (Award-PGRA). This work was part funded by the RCUK Energy Programme (Grant No. EP/I501045). 
*zhisong.qu@anu.edu.au

[1] R. Nazikian et al., Phys. Rev. Lett. 101, 185001 (2008).

[2] T. Ido et al., Nucl. Fusion 51, 073046 (2011).

[3] W. Chen et al., Nucl. Fusion 53, 113010 (2013).

[4] N. Winsor, J. L. Johnson, J. M. Dawson, Phys. Fluids 11, 2448 (1968).

[5] H. Sugama and T.-H. Watanabe, J. Plasma Phys. 72, 825 (2006).

[6] R. K. Fisher, D. C. Pace, G. J. Kramer, M. A. Van Zeeland, R. Nazikian, W. W. Heidbrink, M. García-Muñoz, Nucl. Fusion 52, 123015 (2012).

[7] D. Zarzoso et al., Phys. Rev. Lett. 110, 125002 (2013).

[8] G. Y. Fu, Phys. Rev. Lett. 101, 185002 (2008).

[9] H. Berk and T. Zhou, Nucl. Fusion 50, 035007 (2010).

[10] Z. Qiu, F. Zonca, and L. Chen, Plasma Phys. Controlled Fusion 52, 095003 (2010).

[11] Y. I. Kolesnichenko, B. S. Lepiavko, and V. V. Lutsenko, Plasma Phys. Controlled Fusion 55, 125007 (2013).

[12] L. Wang, J. Q. Dong, Z. He, H. He, and Y. Shen, Phys. Plasmas 21, 072511 (2014).

[13] D. Zarzoso, X. Garbet, Y. Sarazin, R. Dumont, and V. Grandgirard, Phys. Plasmas 19, 022102 (2012).

[14] H. Wang and Y. Todo, Phys. Plasmas 20, 012506 (2013).
[15] H. Wang, Y. Todo, and C. C. Kim, Phys. Rev. Lett. 110, 155006 (2013).

[16] D. Zarzoso, A. Biancalani, A. Bottino, Ph. Lauber, E. Poli, J.-B. Girardo, X. Garbet, and R. J. Dumont, Nucl. Fusion 54, 103006 (2014).

[17] G. F. Chew M. L. Goldberger, and F. E. Low, Proc. R. Soc. A 236, 112 (1956).

[18] Y. I. Kolesnichenko, B. S. Lepiavko, and Y. V. Yakovenko, Plasma Phys. Controlled Fusion 54, 105001 (2012).

[19] R. J. F. Sgalla, A. I. Smolyakov, A. G. Elfimov, and M. F. Bashir, Phys. Lett. A 377, 303 (2013).

[20] J. J. Ramos, Phys. Plasmas 10, 3601 (2003).

[21] J. J. Ramos, Phys. Plasmas 12, 052102 (2005).

[22] J.-B. Girardo, D. Zarzoso, R. Dumont, X. Garbet, Y. Sarazin, and S. Sharapov, Phys. Plasmas 21, 092507 (2014).

[23] A. Hasegawa, Phys. Rev. 169, 204 (1968).

[24] D. Bohm and E. P. Gross, Phys. Rev. 75, 1864 (1949).

[25] A. I. Akhiezer and Y. B. Fainberg, Dokl. Akad. Nauk SSSR, 69, 555 (1949).

[26] C. T. Dum, J. Geophys. Res. 94, 2429 (1989).

[27] T. M. O’Neil and J. H. Malmberg, Phys. Fluids 11, 1754 (1968). 